\title{
Catastrophic Left Ventricular Thrombosis Complicating Extra-corporeal Membrane Oxygenation: A Case Report
}

\author{
Michal Pořízka1, Petr Kopecký1, Vladimír Mikulenka², \\ Jan Kunstýřr , Michal Lipš', Martin Balík ${ }^{1}$ \\ ${ }^{1}$ Department of Anaesthesiology, Resuscitation and Intensive Medicine, \\ First Faculty of Medicine, Charles University and General University Hospital \\ in Prague, Prague, Czech Republic; \\ ${ }^{2} 2^{\text {nd }}$ Department of Surgery - Department of Cardiovascular Surgery, \\ First Faculty of Medicine, Charles University and General University Hospital \\ in Prague, Prague, Czech Republic \\ Received August 21, 2017; Accepted December 5, 2017.
}

Key words: Extra-corporeal membrane oxygenation - Cardiac surgery Thrombosis - Heart failure

Abstract: A massive left ventricular thrombosis represents a rare however, catastrophic complication of a central veno-arterial extra-corporeal membrane oxygenation. We report a case of such complication in a patient with severe left ventricular dysfunction after cardiac surgery. Its management and preventive measures are described and discussed.

The echocardiographic monitoring was supported in part from project reg. no. CZ.2.16/3.1.00/21565 from OP Prague Competitiveness.

Mailing Address: Michal Pořizka, MD., PhD., E.D.I.C., Department of Anaesthesiology, Resuscitation and Intensive Medicine, First Faculty of Medicine, Charles University and General University Hospital in Prague, U Nemocnice 2, 12808 Prague 2, Czech Republic; Mobile Phone: +420 606945 580;

e-mail: rizko@post.cz 


\section{Introduction}

Postcardiotomy heart failure represents a state with excessive morbidity and mortality in cardiac surgery. Veno-arterial extra-corporeal membrane oxygenation (VA-ECMO) is used as a mechanical heart support device when pharmacological inotropic support is ineffective (Fukuhara et al., 2016). Nevertheless, despite its beneficial effect on restoration of hemodynamic stability, there are many serious complications associated with its use. The most common are haemorrhage, severe infection and thromboembolism, all significantly increasing patient's morbidity and mortality (Makdisi and Wang, 2015).

Intracardiac thrombosis associated with VA-ECMO represents a rare, however catastrophic complication in terms of worsening morbidity and mortality related to the risk of systemic thrombus embolization (Williams and Bernstein, 2016). The insufficient unloading of left ventricle (LV) with blood stasis, use of artificial material of ECMO circuit and postoperative procoagulant state represent the most important risk factors for the intracardiac thrombus formation (Makdisi and Wang, 2015). There are only a few case reports describing this complication, thus the evidence for proper management and possible preventive measures is limited (Williams and Bernstein, 2016). Therefore, we present a case report of a patient on postoperative central VA-ECMO who developed a thrombosis of his left ventricular cavity.

\section{Case report}

A seventy-three-year-old male (height $170 \mathrm{~cm}$, weight $68 \mathrm{~kg}$ ) with known long standing history of a moderate aortic stenosis and worsening dyspnoea over the last three months was admitted to the regional hospital with a new onset of chest pain and an episode of pre-syncope. Shortly after the admission he developed a cardiogenic shock with respiratory failure due to pulmonary oedema. Patient had to be intubated and mechanically ventilated. A transoesophageal echocardiography (TEE) revealed a massive aortic regurgitation and severely dysfunctional LV - with ejection fraction of $20 \%$. He was urgently transported to our cardio-vascular centre directly to operating theatre for further surgical management. At the admission patient was in profound circulatory shock state with the need of high vasopressor support (noradrenaline of $1 \mu \mathrm{g} / \mathrm{kg} / \mathrm{min}$ ). Intraoperatively, surgeon replaced severely calcified and degenerated bicuspid aortic valve with aortic bioprosthesis (Carpentier-Edwards Magna Aortic Heart Valve $25 \mathrm{~mm}$, Edwards Lifesciences, USA). No vegetations or other signs of infective endocarditis were found. After the procedure a separation from cardio-pulmonary bypass was unsuccessful due to the extreme LV systolic dysfunction (EF of 10\%) and patient was connected to VA-ECMO (centrifugal pump Maquet Rotaflow RF 32, Maquet Cardiopulmonary AG, Hirrlingen, Germany) with blood flow of $6 \mathrm{l} / \mathrm{min}$. Arterial cannula was placed directly into the ascending aorta without the use of interposed vascular prosthesis and venous drainage cannula into the 


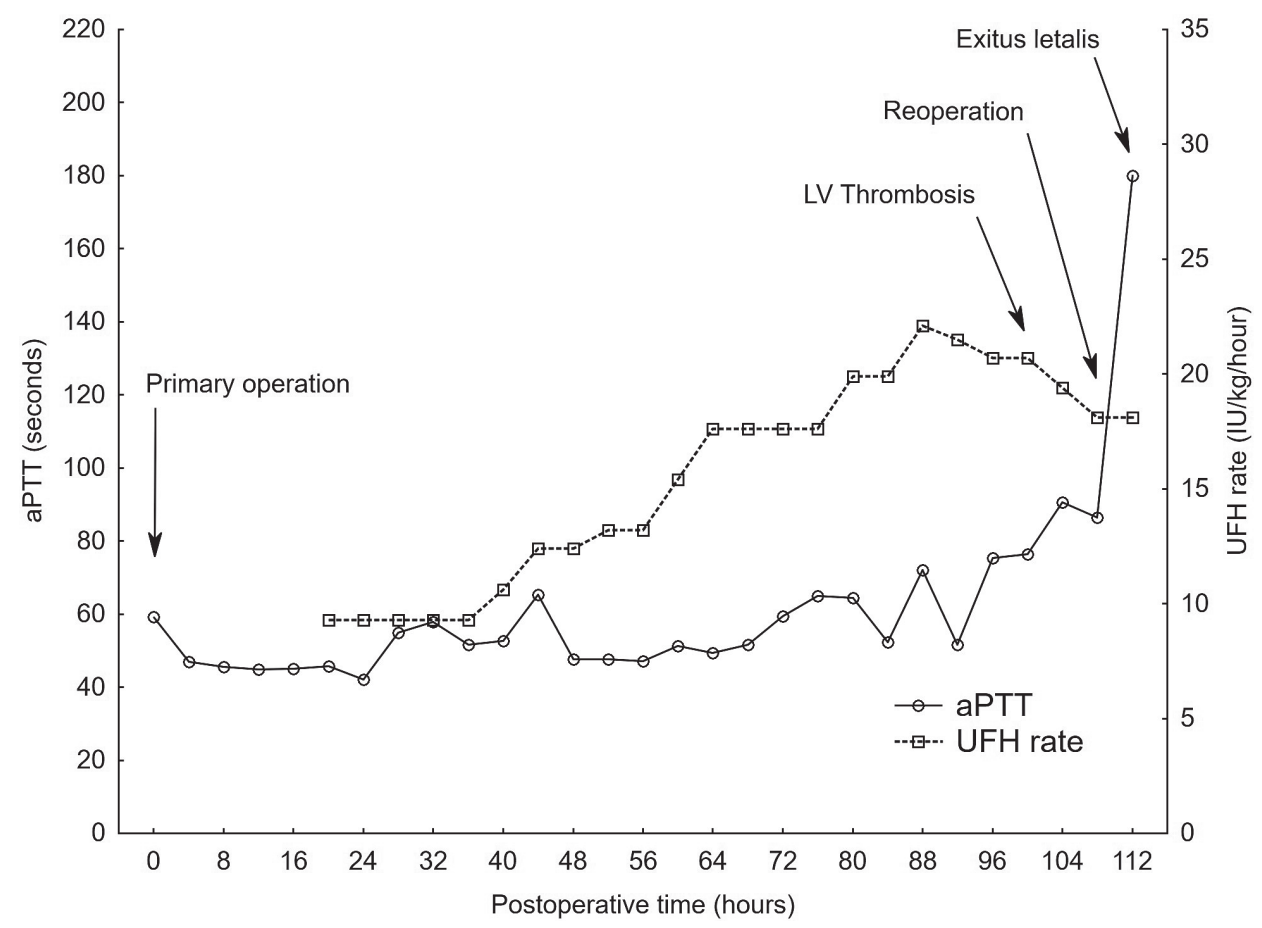

Figure 1 - Unfractionated heparin (UFH) infusion rates and corresponding activated partial thromboplastin times (aPTT) in the postoperative period.

right atrium. Primary sternotomy suture was performed with ECMO tubings placed into the jugular notch. Patient's hemodynamics stabilized on VA-ECMO and noradrenergic support decreased to $0.8 \mu \mathrm{g} / \mathrm{kg} / \mathrm{min}$. There was an excessive blood loss $(650 \mathrm{ml}$ in the first 12 hours postoperatively) due to cardio-pulmonary bypass induced coagulopathy (INR of 1.76), which was normalized by the administration of 6 units of fresh frozen plasma. Simultaneously anaemia was corrected by the transfusion of 3 units of packed red blood cells. Therefore, anticoagulation with unfractionated heparin (UFH) was started on the first postoperative day (POD), when the blood loss was minimal. Infusion rates of UFH and corresponding activated partial thromboplastin times (aPTT) during the postoperative course are depicted in the graph (Figure 1). Patient's clinical status on full VA-ECMO support further improved with continuous decrease of vasopressor requirements. Routine, every-day transthoracic echocardiography (TTE) controls showed a non-dilated, however akinetic left ventricle (LV) without any improvement in contractility even after the administration on inotropes (dobutamine of $10 \mu \mathrm{g} / \mathrm{kg} / \mathrm{min}$ ). Due to the development of tracheobronchitis on the second POD patient had to stay intubated and mechanically ventilated. On the fourth POD the TTE revealed an akinetic, non-dilated LV obliterated with a massive thrombus (Figure 2). Aiming 


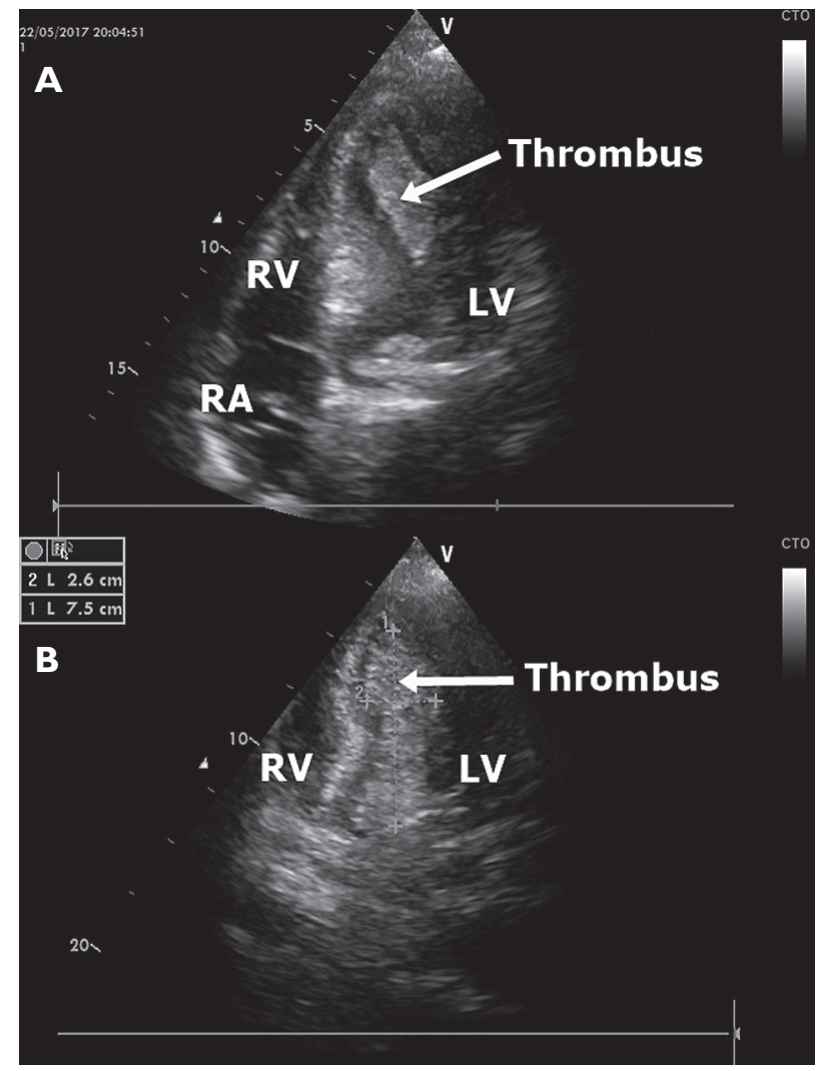

Figure 2 - Transthoracic echocardiographic images of the left ventricular thrombus. $A$ - apical four-chamber view; $B$ - apical five chamber view with the thrombus size measurements ( $L V$ - left ventricle, $R A$ - right atrium, $R V$ - right ventricle).

to evacuate the LV and left atrial (LA) thrombi the patient was reoperated. Intraoperatively other thrombi protruding into the LA from pulmonary veins were found and only partially extracted. The plan was to continue with left heart venting cannula inserted through left atrial appendage and to support contractility with the administration of inotropes. Nevertheless, immediate postoperative TEE was performed and revealed a recurrent massive LV and LA thrombosis. Simultaneously, there was an excessive postoperative bleeding (1000 ml/2 hours) due to severe cardio-pulmonary bypass induced coagulopathy (aPTT of $180 \mathrm{~s}$, INR of 1.5). Patient's prognosis was evaluated as extremely poor and further therapy futile based of the fact of a massive recurrent heart thrombosis, severe coagulopathic bleeding, no LV recovery regardless of the inotropic support on the fifth POD and no eligibility for either long-term circulatory support or heart transplant due to the advanced age. A multi-disciplinary ECMO team decided to limit further therapy and to withdraw VA-ECMO support. Patient's family consented with this approach and patient was disconnected from VA-ECMO. The patient died shortly after the cessation of mechanical heart support. 


\section{Discussion}

Thrombosis of LV cavity represents one of the most serious complications of VA-ECMO. Although it is a relatively common complication in patients with implanted left ventricular assist devices (Reilly et al., 2000), there are only few reports describing ventricular thrombosis in ECMO patients (Gaide-Chevronnay et al., 2012). There are several risk factors for the development of this complication, which were described for both central and peripheral venous cannulation.

The first major risk factor for intra-cardiac clotting is blood stasis in dilated, severely dysfunctional and non-ejecting heart not responding to an inotropic support. Such situation is especially unfavourable and problematic in peripheral VA-ECMO, in which a retrograde aortic blood flow is present, substantially increasing cardiac afterload for LV ejection (Gaide-Chevronnay et al., 2012). However, cases of LV thrombosis in central VA-ECMO with antegrade aortic blood flow have also been reported (Weis et al., 2009). On the other hand, several interventions exist that have the potential to prevent this complication. Firstly, the use of inotropes enhances contractility and augments cardiac ejections and may lead to partial cardiac decompression. This approach often fails in severe myocardial dysfunction, especially in the early phases after ECMO implantation, when unrecovered myocardium may be unresponsive to inotropes. Another way, how to achieve LV unloading, may be by either surgical or less effective percutaneous venting of LV (Meani et al., 2017). Additionally, an indirect LV unloading with intra-aortic balloon pump has been reported with variable success in VA-ECMO patients with partially preserved cardiac contractility (Doll et al., 2004). Finally, very promising possibility is adding Impella device that represents an alternative option to support antegrade flow from LV to aorta (Cheng et al., 2013), however it is not routinely available particularly due to its cost. Nevertheless, due to the insufficient data from clinical trials it is not clear which venting method is superior to another and its effect on mortality also remains unknown (Meani et al., 2017). A different approach compared to VA-ECMO is the use of left-ventricular assist device (LVAD) with the drainage cannula implanted directly into the LV, which has also been successfully used in patients with post-cardiotomy heart failure (Mebazaa et al., 2010). This method enables more efficient drainage of LV cavity compared to VA-ECMO, however fatal intra-cardiac thromboses have been also reported (Reilly et al., 2000). The disadvantages of LVAD in the immediate postoperative period include a need for biventricular support in patients with right ventricular dysfunction or a switch to ECMO circuit with oxygenator in a case of severe respiratory failure. It is also considerably more expensive compared to common VA-ECMO with centrifugal pump. In our institution we use CentriMag Ventricular Assist System (Levitronix, USA) as LVAD. However, in this specific case, the patient was not eligible for heart transplant or long-term LVAD due to the advanced age. Therefore, we decided for VA-ECMO only as a bridge to recovery in a patient who was in critical state with profound low cardiac output syndrome preoperatively, 
thus with high risk of the development of multiple organ failure that is associated with extremely poor prognosis.

The second major risk factor represents a pro-coagulation state induced by systemic inflammatory response syndrome as a reaction to surgery with cardio-pulmonary bypass and ECMO artificial material (tubing, oxygenator). To prevent clotting in the ECMO circuit systemic anticoagulation is mandatory. The most widely used anticoagulation agent for provision of extra-corporeal life support is UFH. It is routinely given as a bolus infusion (50-100 units/kg of body weight) prior to initiation of ECMO and then infused continuously during ECMO support (The Extracorporeal Life Support Organization, 2014). However, no anticoagulation for a short period of time has been suggested in case of major bleeding especially in surgical patients (Marasco et al., 2008). Anticoagulation effect of UFH can be monitored using activated clotting time (ACT), aPTT or antiXa factor levels. The recommended levels of aPTT or ACT are 1.5-2.5 times of baseline values, however specific target values of these parameters for adequate systemic anticoagulation for ECMO patients are not precisely known (Bembea et al., 2013). The desired clinical effect of anticoagulation is also affected by concomitant coagulopathy, thrombocytopenia or hemodilution, which are common in these patients. Therefore, individualized anticoagulation thresholds should be used taking into account underlying coagulopathy, risk of bleeding in surgical patients and risk of ECMO circuit or cardiac thrombosis.

In our patient we started anticoagulation with UFH on the first POD (20 hours after surgery) because there was an excessive surgical bleeding and coagulopathy that had to be corrected in the immediate postoperative period. In the following two days we aimed for aPTT of 55-60 seconds (1.3-1.5 times of our laboratory baseline) due to the risk of surgical bleeding. Later on we targeted for aPTT of 60-80 seconds (1.5-2 times of baseline), which is our routine approach in non-surgical ECMO patients. From the perspective of our case it appears that we should have aimed for the higher aPTT level from the beginning. However, a re-thrombosis of LV developed despite very high level of aPTT (180 s) after reoperation. We did not suspect a heparin resistance as UFH rates were in the recommended range (The Extracorporeal Life Support Organization, 2014) for targeted aPTT levels (Figure 1). We also measured Antithrombin III (ATIII) levels every day, which were in the range of $50-60 \%$ throughout the postoperative course, and we did not supplement it. Due to the insufficient evidence, there is currently no recommended threshold for initiation of ATIII supplementation or desired ATIII levels targets for ECMO patients (The Extracorporeal Life Support Organization, 2014).

Another explanation for a postoperative pro-coagulation state is the development of type II heparin induced thrombocytopenia or presence antiphospholipid syndrome. In the former case, aPTT is artificially prolonged and does not correspond to the true level of anticoagulation. Thus anti-Xa factor 
levels would be a better parameter of anticoagulation monitoring. Nevertheless, we did not screen for pro-coagulation states due to the rapid clinical course after thrombosis occurred. Also no pro-coagulant disorders were known from patient's medical history and no medications with prothrombotic properties were administered to the patient pre- and postoperatively.

The other question is whether we should have vented LV earlier at the time of primary operation. LV venting via left atrium, which we used, is a well described surgical technique in decompressing LV (Weymann et al., 2014). However, it is not our routine approach, because it bears substantial surgical risk in terms of bleeding. We consider this technique only in very high risk patients with signs of significant blood stasis in LV cavity (severe LV dilatation and spontaneous echocardiographic contrast), which were not present at the time of primary operation. Unfortunately, at the end this approach was also ineffective in our patient.

In conclusion, massive LV thrombosis is rare, however devastating complication in patients on central VA-ECMO. Effective decompression of the heart, close monitoring of anticoagulation level and constant vigilance for the detection of prothrombotic states is of paramount importance to prevent its development and subsequent catastrophic consequences.

\section{References}

Bembea, M., Annich, G., Rycus, P., Oldenburg, G., Berkowitz, I., Pronovost, P. (2013) Variability in anticoagulation management of patients on extracorporeal membrane oxygenation: an international survey. Pediatr. Crit. Care Med. 14(2), e77-e84.

Cheng, A., Swartz, M., Massey, H. (2013) Impella to unload the left ventricle during peripheral extracorporeal membrane oxygenation. ASAIO J. 59(5), 533-536.

Doll, N., Kiaii, B., Borger, M., Bucerius, J., Kramer, K., Schmitt, D., Walther, T., Mohr, F. (2004) Five-year results of 219 consecutive patients treated with extracorporeal membrane oxygenation for refractory postoperative cardiogenic shock. Ann. Thorac. Surg. 77, 151-157.

Fukuhara, S., Takeda, K., Garan, A., Kurlansky, P., Hastie, J., Naka, Y., Takayama, H. (2016) Contemporary mechanical circulatory support therapy for postcardiotomy shock. Gen. Thorac. Cardiovasc. Surg. 64(4), 183-191.

Gaide-Chevronnay, L., Durand, M., Rossi-Blancher, M., Bach,V., Chavanon, O., Albaladejo, P. (2012) Cardiac thrombosis in a patient during extracorporeal life support. J. Cardiothorac. Vasc. Anesth. 26(4), 664-665.

Makdisi, G.,Wang, I. (2015) Extra corporeal membrane oxygenation (ECMO) review of a lifesaving technology. J. Thorac. Dis. 7(7), E166-E176.

Marasco, S., Lukas, G., McDonald, M., McMillan, J., Ihle, B. (2008) Review of ECMO (extracorporeal membrane oxygenation) support in critically ill adult patients. Heart Lung Circ. 17, S41-S47.

Meani, P., Gelsomino, S., Natour, E., Johnson, D., Rocca, H., Pappalardo, F., Bidar, E., Makhoul, M., Raffa, G., Heuts, S., Lozekoot, P., Kats, S., Sluijpers, N., Schreurs, R., Delnoij, T., Montalti, A., Sels, J., van de Poll, M., Roekaerts, P., Poels, T., Korver, E., Babar, Z., Maessen, J., Lorusso, R. (2017) Modalities and effects of left ventricle unloading on extracorporeal life support: a review of the current literature. Eur. J. Heart Fail. 19, 84-91 (Suppl. 2).

Mebazaa, A., Pitsis, A., Rudiger, A., Toller, W., Longrois, D., Ricksten, S., Bobek, I., De Hert, S., Wieselthaler, G., Schirmer, U., von Segesser, L., Sander, M., Poldermans, D., Ranucci, M., Karpati, P., Wouters, P., 
Seeberger, M., Schmid, E.,Weder, W., Follath F. (2010) Clinical review: Practical recommendations on the management of perioperative heart failure in cardiac surgery. Crit. Care 14(2), 201.

Reilly, M., Wiegers, S., Cucchiara, A., O'Hara, M., Plappert, T., Loh, E., Acker, M., St. John Sutton, M. (2000) Frequency, risk factors, and clinical outcomes of left ventricular assist device-associated ventricular thrombus. Am. J. Cardiol. 86, 1156-1159.

The Extracorporeal Life Support Organization (ELSO) (2014) ELSO Anticoagulation Guidelines.

Weis, F., Beiras-Fernandez, A., Bruegger, D., Kreth, S., Sodian, R., Kur, F., Weis, M., Nikolaou, K. (2009) Huge intracardiac thrombosis in a patient on veno-arterial extracorporeal membrane oxygenation support. Interact. Cardiovasc. Thorac. Surg. 8(2), 247-249.

Weymann, A., Schmack, B., Sabashnikov, A., Bowles, C., Raake, P., Arif, R., Verch, M., Tochtermann, U., Roggenbach, J., Popov, A., Simon, A., Karck, M., Ruhparwar, A. (2014) Central extracorporeal life support with left ventricular decompression for the treatment of refractory cardiogenic shock and lung failure. J. Cardiothorac. Surg. 9, 60.

Williams, B., Bernstein, W. (2016) Review of venoarterial extracorporeal membrane oxygenation and development of intracardiac thrombosis in adult cardiothoracic patients. J. Extra Corpor. Technol. 48(4), 162-167. 